\title{
Polarization-insensitive Simultaneous Measurement of Torsion and Temperature Using Long-period Fiber Grating Written on Polarization-maintaining Photonic Crystal Fiber
}

\author{
Do Kyung Kim, ${ }^{1}$ Jinsil Han, ${ }^{2}$ and Yong Wook Lee ${ }^{2,3 *}$ \\ ${ }^{1}$ Research Center, PROXIHEALTHCARE Inc., 21 Baekbeom-ro 31-gil, Mapo-gu, Seoul 04147, Korea \\ ${ }^{2}$ Industry 4.0 Convergence Bionics Engineering, Pukyong National University, \\ 45 Yongso-ro, Nam-gu, Busan 48513, Korea \\ ${ }^{3}$ School of Electrical Engineering, Pukyong National University, 45 Yongso-ro, Nam-gu, Busan 48513, Korea
}

(Received January 21, 2021; accepted February 19, 2021)

Keywords: optical fiber sensors, long-period fiber grating, polarization-maintaining photonic crystal fiber, Faraday rotator mirror, torsion, temperature

Here, we report a novel optical fiber grating sensor capable of simultaneous measurement of torsion and temperature by utilizing a long-period fiber grating (LPFG) written on a polarization-maintaining photonic crystal fiber (PMPCF) and a Faraday rotator mirror (FRM) concatenated in series with the LPFG. The LPFG written on the PMPCF, referred to as a PMPC-LPFG, which is connected in series with the FRM, exhibits a polarization-independent wavelength-dependent loss spectrum in its reflection spectrum, which has two attenuation dips (ADs) designated as AD 1 and AD 2. When these two ADs have different cladding-mode orders, they have different torsion responses as well as temperature responses. For the two ADs with resonance wavelengths of $\lambda_{1}=\sim 1508.02 \mathrm{~nm}$ and $\lambda_{2}=\sim 1533.48 \mathrm{~nm}$, torsion and temperature responses were investigated in a twist angle range from -70 to $70^{\circ}$ (step: $10^{\circ}$ ) and an ambient temperature range from 25 to $95{ }^{\circ} \mathrm{C}$ (step: $10{ }^{\circ} \mathrm{C}$ ), respectively. The torsion sensitivities of AD 1 and AD 2 were measured as $\sim 157.0$ and $\sim 272.7 \mathrm{pm} / \mathrm{rad}$ at room temperature, and their temperature sensitivities were measured as $\sim 5.8$ and $\sim 8.2 \mathrm{pm} /{ }^{\circ} \mathrm{C}$, respectively. Owing to their linear and independent responses to torsion and temperature, torsion and temperature changes applied to the PMPC-LPFG can be simultaneously evaluated from the measured wavelength shifts of AD 1 and AD 2 using their predetermined torsion and temperature sensitivities.

\section{Introduction}

Long-period fiber gratings (LPFGs), first developed by Vengsarkar et al., ${ }^{(1)}$ have proven to be able to measure various physical parameters such as strain, ${ }^{(2,3)}$ curvature, ${ }^{(4,5)}$ surrounding refractive index, ${ }^{(6,7)}$ temperature, ${ }^{(8-10)}$ and torsion. ${ }^{(11-13)}$ LPFGs with periodicities of several hundred nanometers are optical fiber devices based on the co-directional mode coupling between the core and cladding modes that satisfy the phase-matching condition, which results in discrete attenuation bands in the transmission spectrum. Changing the above physical parameters applied to an LPFG shifts the resonance wavelengths of these attenuation bands.

*Corresponding author: e-mail: yongwook@pknu.ac.kr https://doi.org/10.18494/SAM.2021.3302 
This perturbation-induced wavelength shift is a fundamental basis of LPFG-based sensors. Although LPFG sensors have intrinsic advantages such as lightweight, compact size, fast response, remote sensing capability, electric passivity, and immunity to electromagnetic interference, they also have one significant limitation: their cross-sensitivity among multiple parameters, for instance, the simultaneous dependence of the attenuation dip (AD) wavelength on torsion and temperature. Thus, minimizing or removing the crosstalk among multiple measurands is critical for measurement accuracy. In recent decades, temperature-independent torsion measurement or the simultaneous measurement of torsion and temperature has been increasingly essential in numerous practical applications, such as vehicle engines, aircraft nacelles, massive engineering structures, and anthropomorphic robots. In terms of LPFG sensors, there have been some approaches proposed for temperature-insensitive torsion measurement ${ }^{(14)}$ or separate measurement of torsion and temperature. ${ }^{(15)}$ By inscribing two different phase-shifted helical LPFGs (referred to as PS-HLPFGs) on a single-mode fiber (SMF) and concatenating them in series, Gao et al. obtained two spectral peaks in their transmission spectrum and utilized them as sensor indicators. ${ }^{(14)}$ Regardless of ambient temperature changes, they could measure the torsion applied to the sensor head by observing the wavelength spacing between these two peaks of the PS-HLPFGs because their wavelength spacing was nearly constant with increasing temperature. However, the sensor output spectrum showed a relatively large insertion loss (IL) ( $20 \mathrm{~dB})$. This IL can deteriorate the signal-to-noise ratio of the sensor. In particular, when the applied torsion increased in a clockwise (CW) or counterclockwise (CCW) direction, the spectral visibility of the sensor indicators decreased. As another approach, Xian et al. suggested cascaded helical LPFGs inscribed on an SMF, designated as cascaded HLPFGs, for the simultaneous measurement of torsion and temperature. ${ }^{(15)}$ By employing cascaded HLPFGs with opposite helicities, they obtained two ADs with different cladding-mode orders in their transmission spectrum. For the $\mathrm{CW}$ or CCW torsion applied to the cascaded HLPFGs, the two ADs moved in opposite directions. As opposed to the torsion response, these two ADs shifted in the same direction with increasing temperature. They utilized two distributedfeedback semiconductor lasers with different lasing wavelengths for sensor interrogation and arranged the laser wavelengths to fall on the wings of the two ADs, whose slopes had opposite signs. Owing to the linear and independent wavelength shifts of the two ADs induced by the applied torsion and temperature changes ( $\Delta \theta$ and $\Delta T$, respectively), they were able to simultaneously estimate $\Delta \theta$ and $\Delta T$.

However, the cladding modes of the PS-HLPFGs and cascaded HLPFGs are keenly sensitive to the bending of the fiber. ${ }^{(16)}$ In other words, bend-induced perturbations can readily affect their transmission spectra and the spectral shapes of their ADs, which results in serious measurement errors. ${ }^{(17)}$ Compared with conventional SMF, a polarization-maintaining photonic crystal fiber (PMPCF) has a tighter mode confinement and a lower effective mode area due to the high refractive index difference between air and silica. These mode characteristics make a PMPCF much more robust to bending. Also, the holey structure of a PMPCF composed of a single material (silica) considerably reduces bend-induced effects. ${ }^{(18)}$ Hence, if we utilize an LPFG inscribed on a PMPCF, referred to as a PMPC-LPFG hereafter, as a sensor head, we can significantly lessen bend-related measurement uncertainty and enhance the 
measurement stability in the simultaneous measurement of torsion and temperature. Here, we present an optical fiber sensor capable of simultaneously measuring torsion and temperature by incorporating a PMPC-LPFG concatenated with a Faraday rotator mirror (FRM). To the best of our knowledge, this is the first report on the simultaneous measurement of torsion and temperature using a PMPC-LPFG ended with an FRM. We fabricated the PMPC-LPFG by scanning the focused beams of $\mathrm{CO}_{2}$ laser pulses on one side of an unjacketed PMPCF at a specific period with a line-by-line technique. The $\mathrm{CO}_{2}$ scanning method is a cost-effective way to inscribe LPFGs compared with other fabrication approaches such as femtosecond laser irradiation $^{(19-21)}$ and wet or dry etching processes. ${ }^{(22-24)}$ For orthogonal input polarization states (IPSs), the transmission spectrum of the fabricated PMPC-LPFG has different ADs, designated as AD 1 and AD 2, with dissimilar cladding-mode orders owing to the PMPCF birefringence. Regardless of the IPS, we can obtain both AD 1 and AD 2 by employing the FRM and connecting it to one end of the PMPC-LPFG. Two combined transmission spectra of the PMPCLPFG, obtained at orthogonal IPSs, appear in the reflection spectrum of the PMPC-LPFG ended with the FRM. Because these two ADs have different cladding-mode orders, their torsion sensitivities are different from each other, and the same is true for their temperature sensitivities. To scrutinize the torsion and temperature responses of $\mathrm{AD} 1$ and $\mathrm{AD} 2$, we applied torsion to the PMPC-LPFG in a twist angle range from -70 to $70^{\circ}$ (step: $10^{\circ}$ ) at room temperature and increased the ambient temperature of the PMPC-LPFG from 25 to $95{ }^{\circ} \mathrm{C}$ (step: $10{ }^{\circ} \mathrm{C}$ ) without any applied torsion. With increasing applied torsion (i.e., $\Delta \theta>0$ ) at room temperature (i.e., $\Delta T=0$ ), $\mathrm{AD} 1$ and $\mathrm{AD} 2$, whose resonance wavelengths are indicated by $\lambda_{1}$ and $\lambda_{2}$, respectively, showed different redshifts. Similarly, with increasing ambient temperature (i.e., $\Delta T>0$ ), $\lambda_{1}$ and $\lambda_{2}$ also showed unequal redshifts. Owing to the linear responses of $\lambda_{1}(\Delta \theta, \Delta T)$ and $\lambda_{2}(\Delta \theta, \Delta T)$ to torsion and temperature and the independence of $\lambda_{1}(\Delta \theta, \Delta T)$ and $\lambda_{2}(\Delta \theta, \Delta T)$, torsion and temperature variations applied to the PMPC-LPFG (i.e., $\Delta \theta$ and $\Delta T$ ) can be simultaneously estimated from the measured wavelength shifts of $\mathrm{AD} 1$ and $\mathrm{AD} 2$ (i.e., $\Delta \lambda_{1}$ and $\Delta \lambda_{2}$ ) using the predetermined torsion and temperature sensitivities of $\lambda_{1}$ and $\lambda_{2}$ (i.e., $\Delta \lambda_{1} / \Delta \theta, \Delta \lambda_{2} / \Delta \theta, \Delta \lambda_{1} / \Delta T$, and $\Delta \lambda_{2} / \Delta T$ ). In the following section, we will describe the fabrication process and spectral characteristics of the sensor head in terms of the PMPC-LPFG only and the PMPC-LPFG concatenated with the FRM. Then, we will provide experimental results on the torsion and temperature responses of the sensor head and some discussion on them. Finally, a summary and conclusion will be given.

\section{Fabrication Process and Spectral Characteristics of Sensor Head}

The mode-field diameters of the PMPCF (Crystal Fibre) used for the inscription of LPFGs are 6.8 and $4.3 \mu \mathrm{m}$ (elliptical core) along its fast and slow axes, respectively. The birefringence and attenuation are $8.18 \times 10^{-4}$ and less than $2 \mathrm{~dB} / \mathrm{km}$ at $1550 \mathrm{~nm}$, respectively. The PMPCF has strong form birefringence and is optimized to generate short beat lengths of less than $4 \mathrm{~mm}$. It is also well known that a PMPCF has reduced bend-induced coupling between orthogonal polarization states. We fabricated the PMPC-LPFG by irradiating $10.6 \mu \mathrm{m} \mathrm{CO}$ laser (Synrad Inc.) pulses onto an unjacketed PMPCF by a line-by-line technique. The grating pitch, grating length, and number of laser scanning cycles of the fabricated PMPC-LPFG were 
$400 \mu \mathrm{m}, 12.0 \mathrm{~mm}$ (30 grating periods), and 4, respectively. The phase-matching condition of a conventional LPFG is given by

$$
\lambda_{\text {res }}=\left(n_{c o, e f f}-n_{c l, e f f}\right) \Lambda,
$$

where $\lambda_{\text {res }}$ is the resonance wavelength, $\Lambda$ is the grating pitch, and $n_{c o, e f f}$ and $n_{c l, e f f}$ are the effective refractive indices of the fundamental core mode and the cladding mode of the LPFG, respectively. Owing to the high birefringence of the PMPCF, caused by its asymmetric cladding structure and the refractive index difference between air and glass, the resonance wavelength satisfying Eq. (1) depends on the IPS. This is because the ratio of the input optical powers coupled to the fast and slow axes of the PMPCF as well as the effective refractive indices ( $n_{c o, e f f}$ and $\left.n_{c l, e f f}\right)$ depend on the IPS. The polarization dependences of $n_{c o, e f f}$ and $n_{c l, e f f}$ result in an input-polarization-dependent distribution of ADs, and two ADs with different claddingmode orders, obtained at orthogonal IPSs, can be found within $100 \mathrm{~nm}$. Figure 1(a) shows the transmission spectra of the fabricated PMPC-LPFG at room temperature with no torsion applied
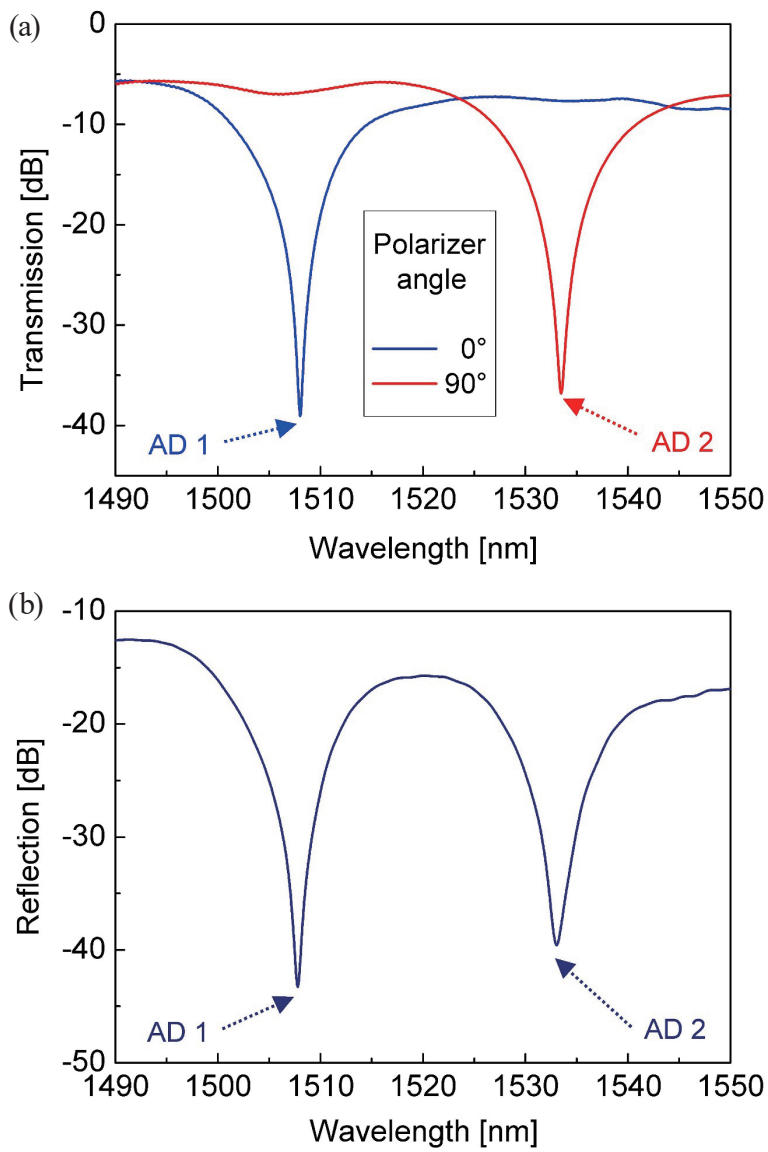

Fig. 1. (Color online) (a) Polarization-dependent transmission spectra of the fabricated PMPC-LPFG, measured for two orthogonal IPSs denoted by polarizer angles of 0 and $90^{\circ}$, indicated as blue and red solid lines, respectively. (b) Measured reflection spectrum of the fabricated PMPC-LPFG concatenated with the FRM. 
to the PMPC-LPFG, measured at two orthogonal IPSs set by a polarization controller (PC) composed of a linear polarizer, a quarter-wave plate (QWP), and a half-wave plate (HWP). To excite the two principal axes of the PMPC-LPFG, we controlled the orientation angles of the polarizer, the QWP, and the HWP, which were contained within the PC. For convenience, we assume that the polarizer angles of 0 and $90^{\circ}$ in Fig. 1(a) stand for these angular arrangements of the PC for exciting the fast and slow axes (or vice versa) of the PMPC-LPFG, respectively. As seen in the figure, we obtained two wavelength-dependent loss dips, indicated by AD 1 (blue) and AD 2 (red) in Fig. 1(a), with maximum band-rejection ratios (BRRs) of $\sim 33.41 \mathrm{~dB}$ at $\lambda_{1}(=\sim 1508.02 \mathrm{~nm})$ and $\sim 31.13 \mathrm{~dB}$ at $\lambda_{2}(=\sim 1533.48 \mathrm{~nm})$ at input polarizer angles of 0 and $90^{\circ}$, respectively. Each $\mathrm{AD}$ with the maximum $\mathrm{BRR}$ is obtained only when input light excites each principal axis of the PMPCF. The BRRs of the fabricated PMPC-LPFG are higher than those of the LPFG inscribed on a conventional polarization-maintaining fiber (referred to as the PMLPFG in the previous work), ${ }^{(25)}$ so the indicator dip becomes sharper, resulting in improved sensing accuracy.

In terms of the PMPC-LPFG connected to the FRM, the FRM plays the role of rotating its IPS by $90^{\circ}$. That is to say, when light emerging from the PMPC-LPFG is reflected from the FRM, the polarization state of light reentering the PMPC-LPFG is a $90^{\circ}$-rotated version of the output polarization state of the PMPC-LPFG. This polarization rotation of the FRM compensates for any birefringence induced during the first propagation of light through the PMPC-LPFG ${ }^{(26)}$ and allows two optical beams passing through the PMPC-LPFG in opposite directions to excite the orthogonal axes of the PMPC-LPFG. Hence, we can determine the reflection spectrum of the PMPC-LPFG by multiplying the two individual transmission spectra obtained when each of its two orthogonal axes is excited [e.g., the two loss spectra indicated as blue and red solid lines in Fig. 1(a)]. In other words, if light entering the PMPC-LPFG couples into one of its principal axes, then the reflected light from the FRM, revisiting the PMPC-LPFG, couples into the other axis. This sequential excitation of the orthogonal principal axes makes the reflection spectrum of the PMPC-LPFG have two resonance dips with the maximum BRRs, indicated as AD 1 and AD 2 in Fig. 1(a). In particular, the reflection spectrum is independent of the input polarization, because one can represent any polarization state as a weighted sum of linear horizontal and vertical polarizations. Owing to this superposition of optical polarization, when the light makes a round trip to the FRM starting from the PMPC-LPFG, incident optical power is equally distributed into both principal axes of the PMPC-LPFG, and thus two resonance dips with the maximum BRRs appear simultaneously in its reflection spectrum regardless of the IPS. ${ }^{(27)}$ The same is true for unpolarized input light, and thus our sensor does not require a polarized light source. The use of an unpolarized light source removes concerns about the measurement instability that can occur due to polarization changes of the input light. Figure 1(b) shows the reflection spectrum of the fabricated PMPC-LPFG concatenated with the FRM, measured using an unpolarized light source. We observed that two wavelengthdependent dips (i.e., AD 1 and AD 2) exist in one reflection spectrum as predicted above. The IL of the reflection spectrum was measured as $\sim 12.55 \mathrm{~dB}$. The measured IL mainly originates from the ILs of the PMPC-LPFG, the FRM, and an optical circulator used for measurement of the reflection spectrum. If $\mathrm{AD} 1$ and $\mathrm{AD} 2$ shown in Fig. 1(b) have dissimilar claddingmode orders, they also have different torsion and temperature sensitivities. ${ }^{(27)}$ By utilizing the 
PMPC-LPFG combined with the FRM, therefore, torsion- and temperature-induced wavelength shifts of both dips, required for the simultaneous measurement of torsion and temperature, can be estimated with only one spectral scan without concern about polarization-change-induced measurement errors.

\section{Experimental Results and Discussion}

Figure 2 shows a schematic diagram of the proposed sensor system incorporating a PMPCLPFG connected with an FRM. We constructed the proposed sensor by using an unpolarized broadband source (BBS, Fiberlabs FL7001), an optical spectrum analyzer (OSA, Yokogawa AQ6370C), an optical circulator (AFW Technologies), the fabricated PMPC-LPFG, and an FRM (OZ Optics). Unpolarized input light enters the PMPC-LPFG via the optical circulator, and light reflected from the FRM goes into the PMPC-LPFG and then the OSA via the optical circulator. To investigate the independent torsion and temperature responses of the fabricated PMPC-LPFG concatenated with the FRM, we prepared an experimental setup by adding a hot plate and a fiber rotator with fiber holders to the constructed sensor, as shown in Fig. 2. In this experimental setup, we monitored the torsion and temperature responses of the PMPC-LPFG with the BBS and the OSA. The optical circulator directs light reflected from the FRM to the OSA and enables the measurement of the reflection spectrum of the PMPC-LPFG concatenated with the FRM. We mounted the fabricated PMPC-LPFG between two fiber holders separated by $L_{0}=200 \mathrm{~mm}$. We fixed one of the two fiber holders and combined the other holder with a fiber rotator to enable it to rotate one end of the PMPC-LPFG. Here, we define the twist direction using the sign of the twist angle: positive and negative twist angles indicate $\mathrm{CW}$ and $\mathrm{CCW}$ torsion applied to the sensor head, as seen towards the fixed fiber holder by the observer, respectively. The twist rate is given by $\theta / L_{0}$, where $\theta$ and $L_{0}$ are the angle of rotation by the fiber rotator and the distance between both fiber holders, respectively. The sensing fiber includes a 44-mm-long PMPCF segment containing a 12-mm-long PMPC-LPFG on its center and two SMF segments fusion-spliced to either end of the PMPCF segment. To minimize bend-induced measurement errors, we applied a certain amount of axial tension to the sensing fiber using a weight of $12.8 \mathrm{~g}(\sim 0.125 \mathrm{~N})$ during the torsion measurement.

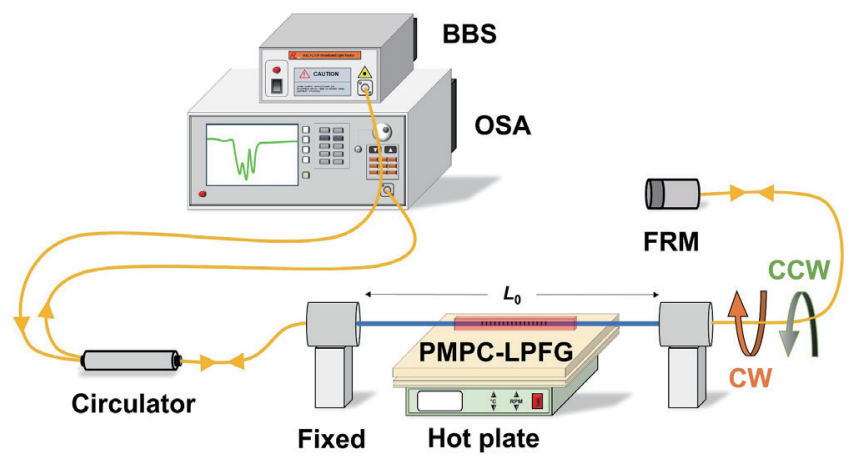

Fig. 2. (Color online) Schematic diagram of the proposed sensor system incorporating a PMPC-LPFG concatenated with an FRM. 
Figures 3(a) and 3(b) show the torsion-induced spectral variations of AD 1 and AD 2 of the PMPC-LPFG concatenated with the FRM, measured for the twist angle variation from -70 to $70^{\circ}$ (step: $10^{\circ}$ ) at room temperature $\left(25^{\circ} \mathrm{C}\right.$ ), respectively. The dashed lines in Fig. 3 represent the measured output reflection spectra with no torsion applied to the PMPC-LPFG. As seen in the figure, each $\mathrm{AD}$ exhibits a redshift or a blueshift for $\mathrm{CW}$ or $\mathrm{CCW}$ torsion, respectively. This torsion-induced wavelength shift can be understood through the photoelastic effect in conjunction with Eq. (1): torsion-induced distortion and shear stress can cause elliptical birefringence due to the photoelastic effect in the $\mathrm{CO}_{2}$-laser-induced LPFG with an asymmetric index profile (i.e., linear birefringence). This torsion-induced elliptical birefringence is proportional to the applied twist rate, and the direction of the elliptical birefringence vector is determined by the twist direction. ${ }^{(28)}$ The torsion-induced right- or left-rotatory elliptical birefringence respectively leads to an increase or decrease, in the difference between $\delta n_{c o, e f f}$

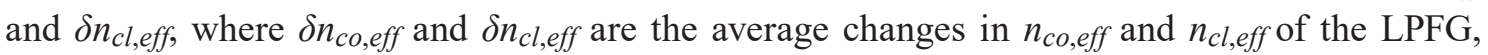
respectively. ${ }^{(28)}$ Hence, when we apply CW or CCW torsion to the PMPC-LPFG, the resonance wavelengths of the ADs of the PMPC-LPFG exhibit a redshift or a blueshift owing to the increase or decrease in $\left(\delta n_{c o, e f f}-\delta n_{c l, e f f}\right)$, respectively. ${ }^{(29)}$ Moreover, the torsion-induced
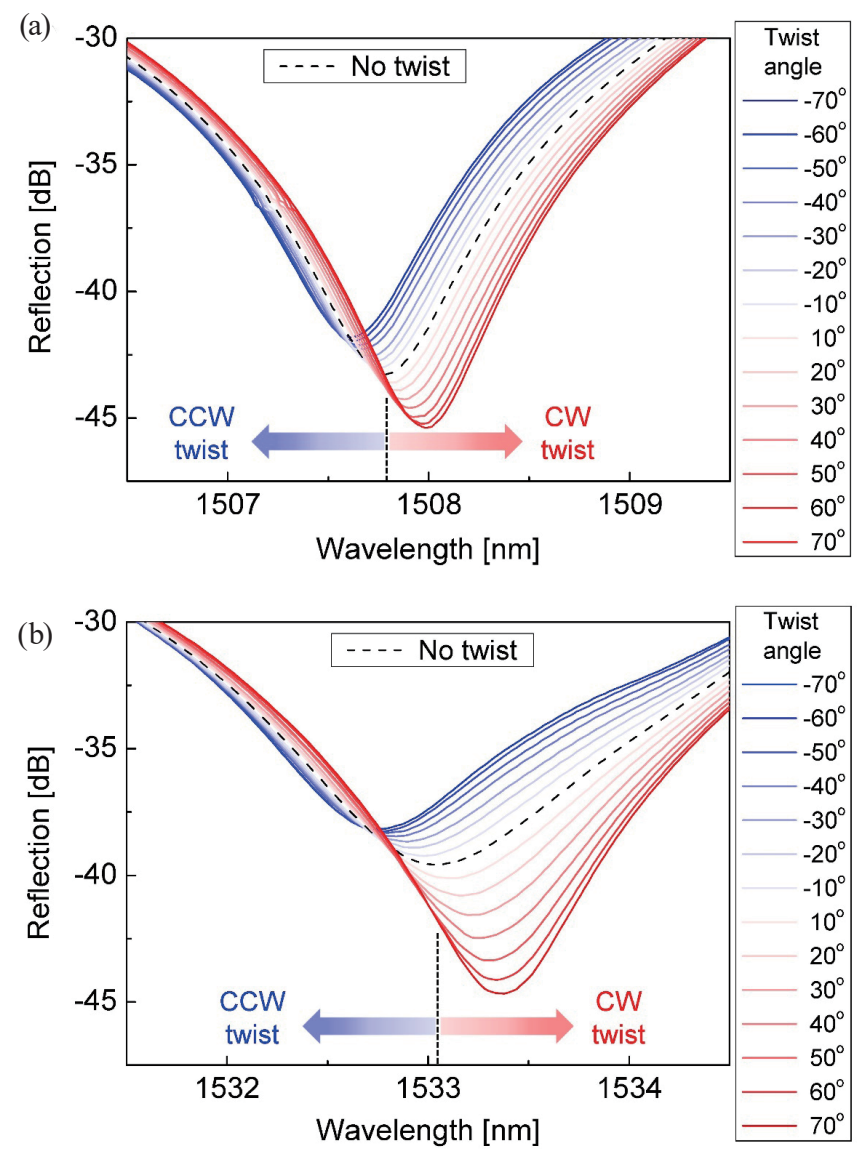

Fig. 3. (Color online) Torsion-induced spectral variations of (a) AD 1 and (b) AD 2 of the PMPC-LPFG concatenated with the FRM measured for the twist angle variation from -70 to $70^{\circ}$ at room temperature $\left(25^{\circ} \mathrm{C}\right)$. The dashed lines represent the measured spectra with no torsion applied to the PMPC-LPFG. 
effective index changes of the cladding, $\delta n_{c l, e f f}$, corresponding to different cladding modes, are dissimilar. Thus, the torsion sensitivity of the resonance wavelength of the LPFG differs depending on the cladding-mode order. ${ }^{(29)} \mathrm{AD} 1$ and $\mathrm{AD} 2$ show total wavelength displacements of 0.38 and $0.64 \mathrm{~nm}$ for a twist angle change of $140^{\circ}$, respectively, which implies that both dips have different cladding-mode orders. Here, the torsion-induced elliptical birefringence in the SMF segment is negligible owing to its nearly circular-symmetric structure and index profile and can be regarded as hardly affecting the IPS of the PMPC-LPFG. ${ }^{(29,30)}$ Moreover, in terms of the sensor head length $L_{0}$ (i.e., the twisted length), the torsion sensitivity defined as $\Delta \lambda / \Delta \theta$ is directly dependent on $L_{0}$ and decreases with increasing $L_{0}$ because $\Delta \lambda$ is proportional to the twist rate. To increase the torsion sensitivity of the sensor, therefore, $L_{0}$ can be decreased to as short as possible but should exceed the length of the PMPC-LPFG.

To examine the thermal responses of AD 1 and AD 2 of the PMPC-LPFG, we placed the entire PMPCF segment (44 mm) containing the PMPC-LPFG on a hot plate, as shown in Fig. 2, and monitored the spectral variations of $\mathrm{AD} 1$ and $\mathrm{AD} 2$ in a temperature range from 25 to $95{ }^{\circ} \mathrm{C}$ (step: $10{ }^{\circ} \mathrm{C}$ ) without applied torsion. During the temperature response test, we used a weight of $12.8 \mathrm{~g}$ on one side of the PMPCF segment with the other side fixed to keep the sensor head straight. Figures 4(a) and 4(b) show the measured temperature-induced spectral
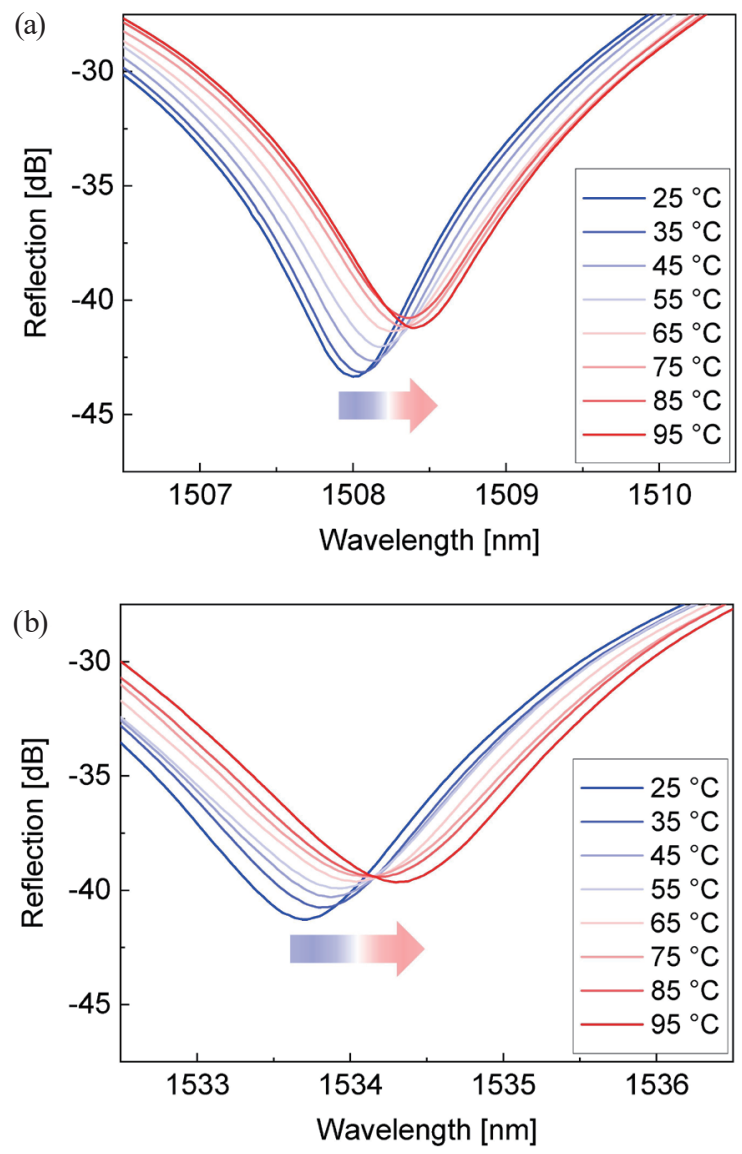

Fig. 4. (Color online) Temperature-induced spectral variations of (a) AD 1 and (b) AD 2 of the PMPC-LPFG concatenated with the FRM, measured for an ambient temperature variation from 25 to $95{ }^{\circ} \mathrm{C}$ without applied torsion. 
variations of AD 1 and AD 2 of the PMPC-LPFG concatenated with the FRM, respectively. As observed in the figure, $\mathrm{AD} 1$ and $\mathrm{AD} 2$ show redshifts (in the direction of the arrows) of 0.40 and $0.60 \mathrm{~nm}$ for a temperature increase of $70{ }^{\circ} \mathrm{C}$, respectively. The thermo-optic coefficient and thermal expansion of the PMPCF are responsible for the temperature-induced spectral shifts, although they are much smaller (specifically up to 30 times smaller) than those of the conventional polarization-maintaining fiber. Figure 5(a) shows the torsion-induced resonance wavelength shifts of AD 1 and AD 2 of the PMPC-LPFG concatenated with the FRM, indicated by blue circular symbols and red rectangular symbols, respectively. Blue and red solid lines indicate the linear fits of the measured data points of $\mathrm{AD} 1$ and $\mathrm{AD} 2$, respectively. It is found from this result that $\mathrm{AD} 1$ and $\mathrm{AD} 2$ show highly linear torsion responses with adjusted $R^{2}$ values of $\sim 0.99775$ and $\sim 0.99485$, respectively. The torsion sensitivities of AD 1 and AD 2 were evaluated as $\Delta \lambda_{1} / \Delta \theta=\sim 157.0 \mathrm{pm} / \mathrm{rad}$ and $\Delta \lambda_{2} / \Delta \theta=\sim 272.7 \mathrm{pm} / \mathrm{rad}$, respectively. The torsion sensitivity of $\mathrm{AD} 2$ is about 1.7 times greater than that of $\mathrm{AD} 1$, which means that the ADs have different torsion responses, that is, $\lambda_{1}(\Delta \theta, \Delta T=0)$ is independent of $\lambda_{2}(\Delta \theta$, $\Delta T=0$ ). Similarly, Fig. 5(b) shows the temperature-induced resonance wavelength shifts of AD 1 and AD 2 of the PMPC-LPFG concatenated with the FRM, indicated by blue circular
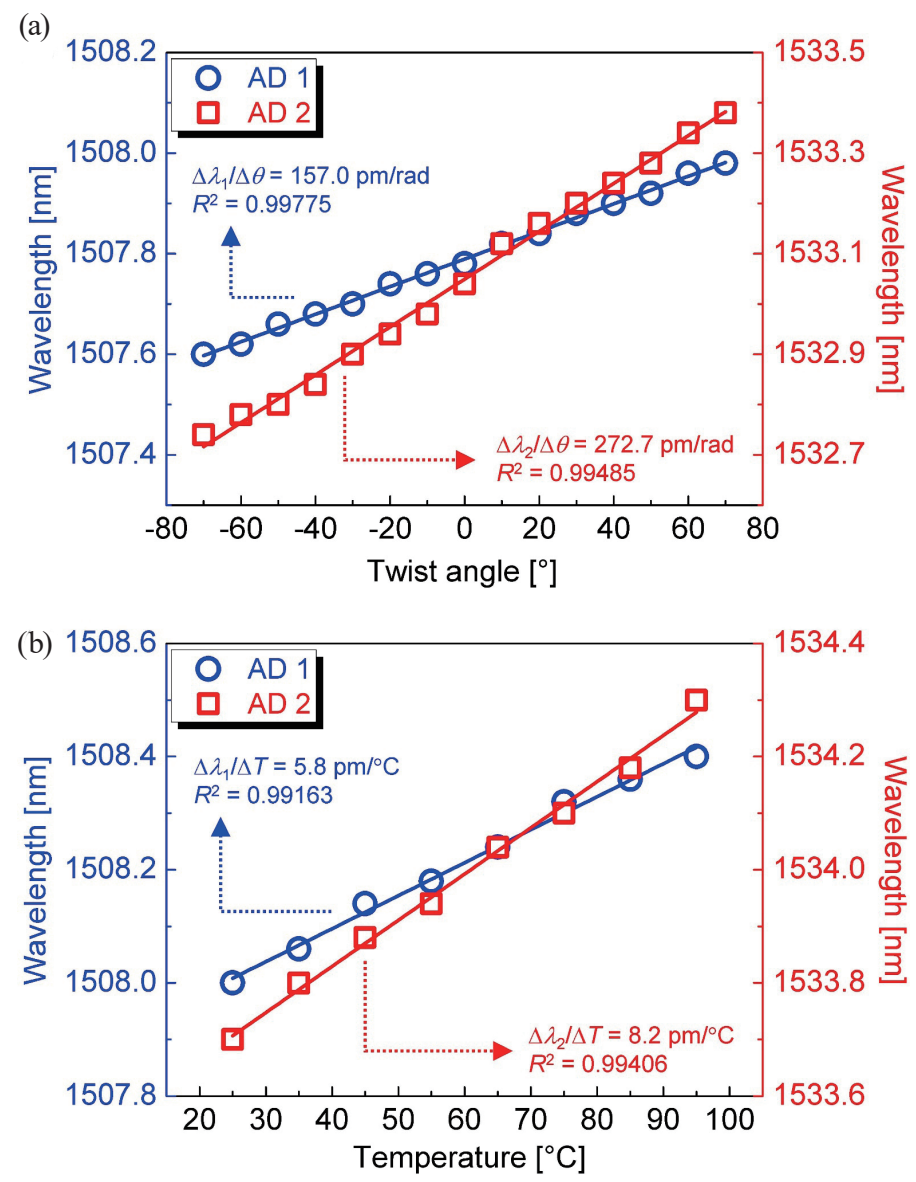

Fig. 5. (Color online) (a) Torsion- and (b) temperature-induced resonance wavelength shifts of AD 1 and AD 2 of the PMPC-LPFG concatenated with the FRM, indicated by blue circular symbols and red rectangular symbols, respectively. Blue and red solid lines indicate the linear fits of measured data points of AD 1 and AD 2, respectively. 
symbols and red rectangular symbols, respectively. Similarly to in Fig. 5(a), blue and red solid lines represent the linear fits of the measured data points of $\mathrm{AD} 1$ and $\mathrm{AD} 2$, respectively. The calculated temperature sensitivities of $\mathrm{AD} 1$ and $\mathrm{AD} 2$ were $\Delta \lambda_{1} / \Delta T=\sim 5.8 \mathrm{pm} /{ }^{\circ} \mathrm{C}$ and $\Delta \lambda_{2} / \Delta T=\sim 8.2 \mathrm{pm} /{ }^{\circ} \mathrm{C}$ with adjusted $R^{2}$ values of $\sim 0.99163$ and $\sim 0.99406$, exhibiting the good linearity of the temperature response, respectively. The difference in the temperature sensitivity tells us that $\lambda_{1}(\Delta \theta=0, \Delta T)$ is uncorrelated with $\lambda_{2}(\Delta \theta=0, \Delta T)$. These temperature sensitivities of the PMPC-LPFG are much smaller than those of the PM-LPFG $\left(129.12\right.$ and $\left.101.08 \mathrm{pm} /{ }^{\circ} \mathrm{C}\right) .(25,27)$ The single material property of the PMPCF made of pure silica is responsible for this small temperature dependence. However, the low temperature sensitivity does not impair the stability of simultaneous measurements of torsion and temperature. Rather, the temperature measurement range of the PMPC-LPFG can be broadened at the expense of the temperature sensitivity in comparison with the measurement based on the PM-LPFG because the BBS used as an input light source has a finite spectral bandwidth. It is concluded from Fig. 5 that the two resonance dips (i.e., AD 1 and AD 2) of the PMPC-LPFG have linear and independent responses to torsion and temperature.

The linearities of $\lambda_{1}(\Delta \theta, \Delta T)$ and $\lambda_{2}(\Delta \theta, \Delta T)$ and their independence make it possible to simultaneously measure changes in applied torsion and temperature ( $\Delta \theta$ and $\Delta T$, respectively). If we designate the torsion and temperature sensitivities of the two ADs as four sensitivity coefficients $C_{1}\left(=\Delta \lambda_{1} / \Delta \theta\right), C_{2}\left(=\Delta \lambda_{1} / \Delta T\right), C_{3}\left(=\Delta \lambda_{2} / \Delta \theta\right)$, and $C_{4}\left(=\Delta \lambda_{2} / \Delta T\right)$, these coefficients can be directly utilized for the simultaneous measurement of torsion and temperature variations $(\Delta \theta$ and $\Delta T$ ) applied to the PMPC-LPFG through Eq. (2).

$$
\left[\begin{array}{c}
\Delta \lambda_{1} \\
\Delta \lambda_{2}
\end{array}\right]=\left[\begin{array}{ll}
C_{1} & C_{2} \\
C_{3} & C_{4}
\end{array}\right]\left[\begin{array}{l}
\Delta \theta \\
\Delta T
\end{array}\right]
$$

According to the predetermined sensitivities obtained above, $C_{1}=\sim 157.0 \mathrm{pm} / \mathrm{rad}$, $C_{2}=\sim 5.8 \mathrm{pm} /{ }^{\circ} \mathrm{C}, C_{3}=\sim 272.7 \mathrm{pm} / \mathrm{rad}$, and $C_{4}=\sim 8.2 \mathrm{pm} /{ }^{\circ} \mathrm{C}$. On the basis of these four sensitivity coefficients, by measuring $\Delta \lambda_{1}$ and $\Delta \lambda_{2}$, induced by $\Delta \theta$ and $\Delta T$ applied to the PMPCLPFG connected to the FRM, from its reflection spectrum, $\Delta \theta$ and $\Delta T$ can be calculated from Eq. (3).

$$
\left[\begin{array}{c}
\Delta \theta \\
\Delta T
\end{array}\right]=\left[\begin{array}{ll}
157.0 & 5.8 \\
272.7 & 8.2
\end{array}\right]^{-1}\left[\begin{array}{l}
\Delta \lambda_{1} \\
\Delta \lambda_{2}
\end{array}\right]
$$

In brief, our sensor system can provide the simultaneous measurement capability of torsion and temperature by harnessing the linear and uncorrelated torsion and temperature responses of the fabricated PMPC-LPFG. Our sensor system can perform the polarization-insensitive simultaneous measurement of torsion and temperature, which is robust to polarization changes induced by environmental perturbations. 


\section{Conclusion}

We experimentally demonstrated an optical fiber sensor capable of discriminating between torsion and temperature by incorporating a PMPC-LPFG concatenated with an FRM. The PMPC-LPFG as a sensor head was fabricated by scanning $\mathrm{CO}_{2}$ laser pulses on a PMPCF with a specific period. The fabricated PMPC-LPFG connected in series with the FRM exhibited a polarization-independent wavelength-dependent loss spectrum in its reflection spectrum, which has two ADs (AD 1 and AD 2) with different cladding-mode orders. The torsion and temperature responses of these two ADs were investigated in a twist angle range from -70 to $70^{\circ}$ and a temperature range from 25 to $95{ }^{\circ} \mathrm{C}$. The two dips showed linear and independent responses to the applied torsion and temperature, and their torsion and temperature sensitivities were measured as $\sim 157.0 \mathrm{pm} / \mathrm{rad}$ and $\sim 5.8 \mathrm{pm} /{ }^{\circ} \mathrm{C}$ for $\mathrm{AD} 1$ and $\sim 272.7 \mathrm{pm} / \mathrm{rad}$ and $\sim 8.2 \mathrm{pm} /{ }^{\circ} \mathrm{C}$ for $\mathrm{AD} 2$, respectively. With these four sensitivity coefficients, torsion and temperature changes applied to the PMPC-LPFG can be simultaneously estimated from the measured wavelength shifts of the two ADs (AD 1 and AD 2). This implies that the PMPC-LPFG combined with the FRM can be used as an efficient polarization-insensitive sensor head for the single-scan simultaneous sensing of torsion and temperature.

\section{Acknowledgments}

This research was supported by Korea Institute for Advancement of Technology (KIAT) grant funded by the Korea Government (MOTIE) (P0012451, The Competency Development Program for Industry Specialist) and the Basic Science Research Program through the National Research Foundation of Korea (NRF) funded by the Ministry of Education (2019R1I1A3A01046232).

\section{References}

1 A. M. Vengsarkar, P. J. Lemaire, J. B. Judkins, V. Bhatia, T. Erdogan, and J. E. Sipe: J. Lightwave Technol. 14 (1996) 58. http://doi.org/10.1109/50.476137

2 Y. P. Wang, L. Xiao, D. N. Wang, and W. Jin: Opt. Lett. 31 (2006) 3414. http://doi.org/10.1364/OL.31.003414

3 Y. Huang, Z. Zhou, Y. Zhang, G. Chen, and H. Xiao: IEEE Trans. Instrum. Meas. 59 (2010) 2997. http://doi. org/10.1109/TIM.2010.2047065

4 Y. Liu, J. A. R. Williams, and I. Bennion: IEEE Photonics Technol. Lett. 12 (2000) 531. http://doi. org $/ 10.1109 / 68.841276$

5 Y. P. Wang and Y. J. Rao: IEEE Sens. J. 5 (2005) 839. http://doi.org/10.1109/JSEN.2005.844335

6 K. S. Chiang, Y. Liu, M. N. Ng, and X. Dong: Electron. Lett. 36 (2000) 966. http://doi.org/10.1049/el:20000701

7 L. Qi, C.-L. Zhao, J. Yuan, M. Ye, J. Wang, Z. Zhang, and S. Jin: Sens. Actuator, B 193 (2014) 185. http://doi. org/10.1016/j.snb.2013.11.063

8 X. Shu, T. Allsop, B. Gwandu, L. Zhang, and I. Bennion: IEEE Photonics Technol. Lett. 13 (2001) 818. http:// doi.org/10.1109/68.935814

9 Y. P. Wang, D. N. Wang, and W. Jin: Appl. Opt. 45 (2006) 7966. http://doi.org/10.1364/AO.45.007966

10 Q. Wang, C. Du, J. Zhang, R. Lv, and Y. Zhao: Opt. Commun. 377 (2016) 89. http://doi.org/10.1016/ j.optcom.2016.05.039

11 D. E. Ceballos-Herrera, I. Torres-Gomez, A. Martinez-Rios, L. García, and J. J. Sánchez-Mondragón: IEEE Sens. J. 10 (2010) 1200. http://doi.org/10.1109/JSEN.2010.2042951

12 Y. J. Rao, T. Zhu, and Q. J. Mo: Opt. Commun. 266 (2006) 187. http://doi.org/10.1016/j.optcom.2006.04.045

13 M. Deng, J. Xu, Z. Zhang, Z. Bai, S. Liu, Y. Wang, Y. Zhang, C. Liao, W. Jin, G. Peng, and Y. Wang: Opt. Express 25 (2017) 14308. http://doi.org/10.1364/OE.25.014308 
14 R. Gao, Y. Jiang, and L. Jiang: Opt. Express 22 (2014) 15697. http://doi.org/10.1364/OE.22.015697

15 L. Xian, P. Wang, and H. Li: Opt. Express 22 (2014) 20260. http://doi.org/10.1364/OE.22.020260

16 B. H. Lee and J. Nishii: Opt. Lett. 23 (1998) 1624. http://doi.org/10.1364/OL.23.001624

17 N. H. Vu, I. K. Hwang, and Y. H. Lee: Opt. Lett. 33 (2008) 119. http://doi.org/10.1364/OL.33.000119

18 H. Ademgil, S. Haxha, and F. AbdelMalek: Engineering 2 (2010) 608. http://doi.org/10.4236/eng.2010.28078

19 Y. Kondo, K. Nouchi, T. Mitsuyu, M. Watanabe, P. G. Kazansky, and K. Hirao: Opt. Lett. 24 (1999) 646. http://doi.org/10.1364/OL.24.000646

20 A. I. Kalachev, D. N. Nikogosyan, and G. Brambilla: J. Lightwave Technol. 23 (2005) 2568. http://doi. org/10.1109/JLT.2005.851335

21 B. Li, L. Jiang, S. Wang, H.-L. Tsai, and H. Xiao: Opt. Laser Technol. 43 (2011) 1420. http://doi.org/10.1016/ j.optlastec.2011.04.011

22 C.-C. Chiang, T.-C. Cheng, H.-J. Chang, and L. Tsai: Opt. Lett. 34 (2009) 3677. http://doi.org/10.1364/ OL. 34.003677

23 C.-C. Chiang, H.-J. Chang, and J.-S. Kuo: J. Micro-Nanolithogr. MEMS MOEMS 9 (2010) 033007. http://doi. org/10.3390/ma10111297

24 C.-C. Chiang and L. Tsai: Opt. Lett. 37 (2012) 193. http://doi.org/10.1364/OL.37.000193

25 K. J. Han, Y. W. Lee, J. Kwon, S. Roh, J. Jung, and B. Lee: IEEE Photonics Technol. Lett. 16 (2004) 2114. http://doi.org/10.1109/LPT.2004.833081

26 M. Martinelli: Opt. Commun. 72 (1989) 341. http://doi.org/10.1016/0030-4018(89)90436-7

27 Y. W. Lee, K. J. Han, J. Jung, B. Lee, B.-J. Kim, B.-G. Chae, and H.-T. Kim: Jpn. J. Appl. Phys. 45 (2006) L92. http://doi.org/10.1143/JJAP.45.L92

28 R. Ulrich and A. Simon: Appl. Opt. 18 (1979) 2241. http://doi.org/10.1364/AO.18.002241

29 Y. P. Wang, J. P. Chen, and Y. J. Rao: J. Opt. Soc. Am. B-Opt. Phys. 22 (2005) 1167. http://doi.org/10.1364/ JOSAB.22.001167

30 C. L. Zhao, X. Yang, C. Lu, W. Jin, and M. S. Demokan: IEEE Photonics Technol. Lett. 16 (2004) 2535. http:// doi.org/10.1109/LPT.2004.835646

\section{About the Authors}

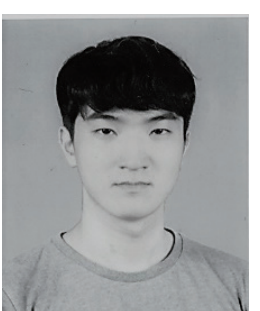

Do Kyung Kim received his B.S. and M.S. degrees from the School of Electrical Engineering and the Interdisciplinary Program of Biomedical, Mechanical and Electrical Engineering, Pukyong National University, Busan, South Korea in 2017 and 2019, respectively. He is currently a researcher in PROXIHEALTHCARE. His research interests include optical fiber devices for optical sensors and filters. (dosokre@naver.com)

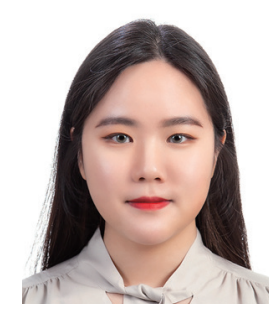

Jinsil Han received her B.S. degree from the School of Electrical Engineering, Pukyong National University, Busan, South Korea, in 2020, where she is currently pursuing her master's degree under Industry 4.0 Convergence Bionics Engineering. Her research interests include fiber-optic physical, chemical, and biosensors. (jinsil3208@pukyong.ac.kr)

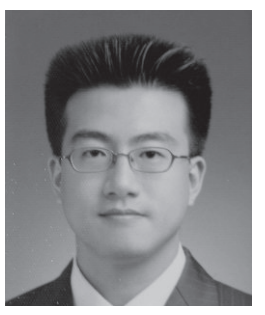

Yong Wook Lee received his B.S., M.S., and Ph.D. degrees from the School of Electrical Engineering, Seoul National University, Seoul, South Korea, in 1998, 2000, and 2004, respectively. From 2004 to 2008, he was a senior researcher in the Electronics and Telecommunications Research Institute, Daejeon, South Korea. He is currently a professor in the School of Electrical Engineering, Pukyong National University, Busan, South Korea. He is the author of more than 120 journal papers. His research interests include the areas of optical devices for optical sensors and communications, such as optical fiber gratings, optical filters, optical amplifiers, and optical switching in semiconductor thin films based on photoinduced phase transition. (yongwook@pknu.ac.kr) 